Int. J. Dev. Biol. 58: 669-676 (2014)

doi: $10.1387 / \mathrm{ijdb} .140174 \mathrm{ss}$

\title{
Cloning, expression and characterization of the ornithine decarboxylase gene from Dictyostelium discoideum
}

\author{
RISHIKESH KUMAR, SHEIKH RAFIA and SHWETA SARAN* \\ School of Life Sciences, Jawaharlal Nehru University, New Delhi, India.
}

\begin{abstract}
Ornithine decarboxylase (ODC) is a rate limiting enzyme in polyamine synthesis that decarboxylates ornithine to form the diamine putrescine. We report here the isolation, expression and characterization of a homolog of ODC from Dictyostelium discoideum. DdODC is conserved and shows sequence and structural homology with that from human. Both ODC transcript and protein are expressed at all stages of development and show high expression in prestalk/stalk cells. It is cytosolic and predominantly perinuclear in localization. Both overexpression of DdODC and putrescine treatment resulted in inhibition of cell proliferation.
\end{abstract}

KEY WORDS: $D$. discoideum, ODC expression, putrescine

Polyamines are ubiquitously present from bacteria to mammals (Tabor and Tabor 1985, Janne 2004) and regulate cell growth, proliferation, differentiation and cell death. The fast proliferating prokaryotes mostly contain putrescine and spermidine (Tabor and Tabor 1976) while the slow proliferating eukaryotes predominantly have spermidine and spermine (Fillingame 1975). A decrease in intracellular polyamines occurs during aging (Minois et al., 2011) but it still remains unclear whether this is a cause or consequence of aging. Addition of exogenous spermidine can extend lifespan in various animal models through epigenetic modifications, induction of autophagy and suppression of necrosis (Eisenberg et al., 2009).

Ornithine decarboxylase (ODC, EC 4.1.1.17) catalyses the first and the rate limiting step of polyamine biosynthesis where Lornithine is converted to putrescine (Heller et al., 1976). It is strictly regulated during development (Heby and Persson 1990) and is involved in synthesis of spermidine and spermine. Decarboxylation of ornithine by ODC is the only pathway for de novo synthesis of polyamines in mammals and higher eukaryotes. The ability to strictly regulate cellular polyamine levels within a very narrow range is critical since extreme levels of polyamines (high or low) result in damaging effects, both on life of the cell and organism as a whole (Pegg and McCann 1982).

This study was undertaken to understand the role of putrescine, which is the most abundant polyamine found in Dictyostelium discoideum (Saran 1998). D. discoideum, a lower eukaryote has both unicellular and multicellular stages in its life cycle (Bonner 1944). Nutrient plays an important role and starvation triggers the vegetative amoebae to enter multicellularity. Vegetative amoeba grow and divide mitotically but upon starvation and in response to the chemoattractant, cAMP, they enter the developmental phase to ultimately form a fruiting body consisting of dead vacuolated stalk cells and viable spores. Since ODC is responsible for the formation of putrescine we characterized this enzyme. Harris and North (1982) did not find any significant ODC activity during development but Campagne and Lowik (1985) have reported high activity at the slug stage. Our earlier report (Saran 1998) has shown high putrescine levels during slug stage.

This is the first report on molecular cloning, expression and characterization of a putative ODC-like sequence from $D$. discoideum. We found it to be structurally conserved and predominantly expressed in the prestalk/stalk cells of multicellular structures. ODC fusion protein was localized in the cytosol in a large vacuole-like structure whose identity is still unclear. Both ODC overexpression $\left(O D C^{O E}\right)$ and putrescine treatment slow growth. Putrescine treatment caused a block in the G2/M phase of cell cycle. Our results show putrescine to be more involved in cell proliferation than development.

\section{Results and Discussion}

Polyamines are essential for various processes of life but their role in either growth or development has been difficult to analyze

Abbreviations used in this paper: DdODC, Dictyostelium discoideum ODC; ODC, ornithine decarboxylase.

*Address correspondence to: Shweta Saran. School of Life Sciences, Jawaharlal Nehru University, New Delhi- 110 067, India. Tel: +91-11-2670-4157.

E-mail: ssaran@mail.jnu.ac.in

Supplementary Material (three figures) for this paper is available at: http://dx.doi.org/10.1387/ijdb.140174ss

Accepted: 24 September 2014.

ISSN: Online 1696-3547, Print 0214-6282

(C) 2014 UBC Press

Printed in Spain 
A

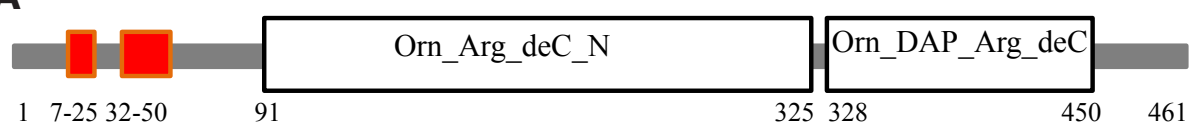

B

D.dis

H. sap

M.mus

D.dis

H.sap

M. mus

D.dis

H. sap

M. mus

D.dis

H.sap

M.mus

D.dis

H. sap

M. mus

D.dis

H. sap

M.mus

D.dis

H. sap

M.mus

D.dis

H.sap

M. mus

D.dis

H.sap

M.mus

MTGTKRNGEEVVNENNNNNVAEETNKKAKVDESSTETTESTSCSLLSRCEKLDIVRKELD $-----------------------------------------M$ MNNFGNEEFD $--------------------------------------------M S S F T K D E F D$ . : .* : * VKPWDQG---KVTIQELITSLLDKTDRDAFFVADVGVIIKQWQKWVKNLPNVKPYYAVKC CHFLDEGFTAKDI LDQKINEVSSSDDKDAFYVADLGDI LKKHLRWLKALPRVTPFYAVKC CHILDEGFTAKDILDQKINEVSSSDDKDAFYVADLGDILKKHLRWLKALPRVTPFYAVKC

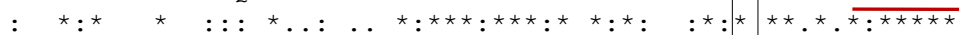
NPTVGVLRVLDALGTNYDCASRTEIESVLNLGVDPSRI IYANPCKQISALKFARAHNVKL NDSKAIVKTLAATGTGFDCASKTEIQLVQSLGVPPERI IYANPCKQVSQIKYAANNGVQM NDSRAIVSTLAAIGTGEDCASKTEIQLVQGLGVPAERVIYANPCKQVSQIKYAASNGVQM

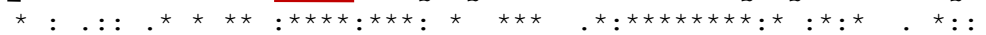
MTFDNLSELEKIEKFFPEAELVLRIAPDDSKSVMRFGSKFGVHIDDCNDLLEMAKEMNLK MTFDSEVELMKVARAHPKAKLVLRIATDDSKAVCRLSVKFGATLRTSRLLLERAKELNID MTFDSE IELMKVARAHPKAKLVLRIATDDSKAVCRLSVKFGATLKTSRLLLERAKELNID

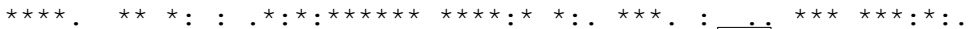
VVGVSFHVGSGCQSGDSYADALIMVKSVFDMAKKLNMELTLVDVEGGHTGSDDEKF--NA VVGVSFHVGSGCTDPETFVQAISDARCVFDMGAEVGFSMYLLDIGGGHPGSEDVKLKFEE VIGVSFHVGSGCTDPETFVQAVSDARCVFDMATEVGFSMHLLDIGGGEPGSEDTKLKFEE

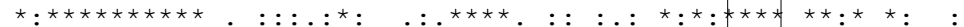
FTKVIREKTAELFS--PNVKI IAEPGRYFAAQSHTLAVTVISKRS IKQEDNRQHPRRTSN ITGVINPALDKYFPSDSGVRI IAEPGRYYVASAFTLAVNI IAKKIVLKEQTGSDD-EDES ITSVINPALDKYFPSDSGVRI IAEPGRYYVASAFTLAVNI IAKKTVWKEQPGSDD-EDES

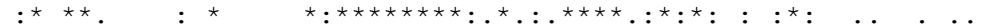
NMRQYNYYLADGVYGSENNTKEDYAKVEPLLLKPSTK--QPTPCTLFGPTCDSIDVVLKD SEQTFMYYVNDGVYGSFNCILYDHAHVKPLLQKRPKPDEKYYSSS IWGPTCDGLDRIVER NEQTFMYYVNDGVYGSFNCILYDHAHVKALLQKRPKPDEKYYSSS IWGPTCDGLDRIVER

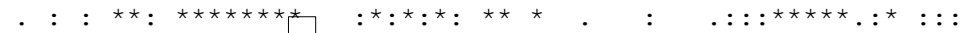
TQIPELKIGDWLYFQDMGAYTIASSSSFNGFCPPPVYYYNSIPEEELKNL---------- 461 CDLPEMHVGDWMLFENMGAYTVAAASTFNGFQRPT IYYVMSGPAWQLMQQFQNPDFPPEV CNLPEMHVGDWMLFENMGAYTVAAAST FNGEQRPNIYYVMSRPMWQLMKQIQSHGFPPEV

$$
::^{\star \star}:::^{\star \star \star}:{ }^{\star}::_{\star \star \star \star \star \star}:{ }^{\star}::^{\star}:{ }^{\star \star \star \star} \quad{ }^{\star \star \star}{ }^{\star \star}:^{*}:
$$

EEQDASTLPVSCAWESGMKRHRAACASAS INV EEQDDGTLPMSCAQESGMDRHPAACASARINV
60

\section{Fig. 1. Domain architecture and multiple} alignment of Dictyostelium discoideum ornithine decarboxylase (DdODC). (A) Domain architecture of DdODC as predicted by Pfam programme. Small red bars represent low complexity regions within DdODC. (B) Multiple sequence alignment of full length ODCs from human (Hs), mouse $(\mathrm{Mm})$ and D. discoideum (Dd). Red letters represent amino acids constituting Orn_Arg_deC-N domain and blue letters show Orn_DAP_Arg_deC domain. See text for details.

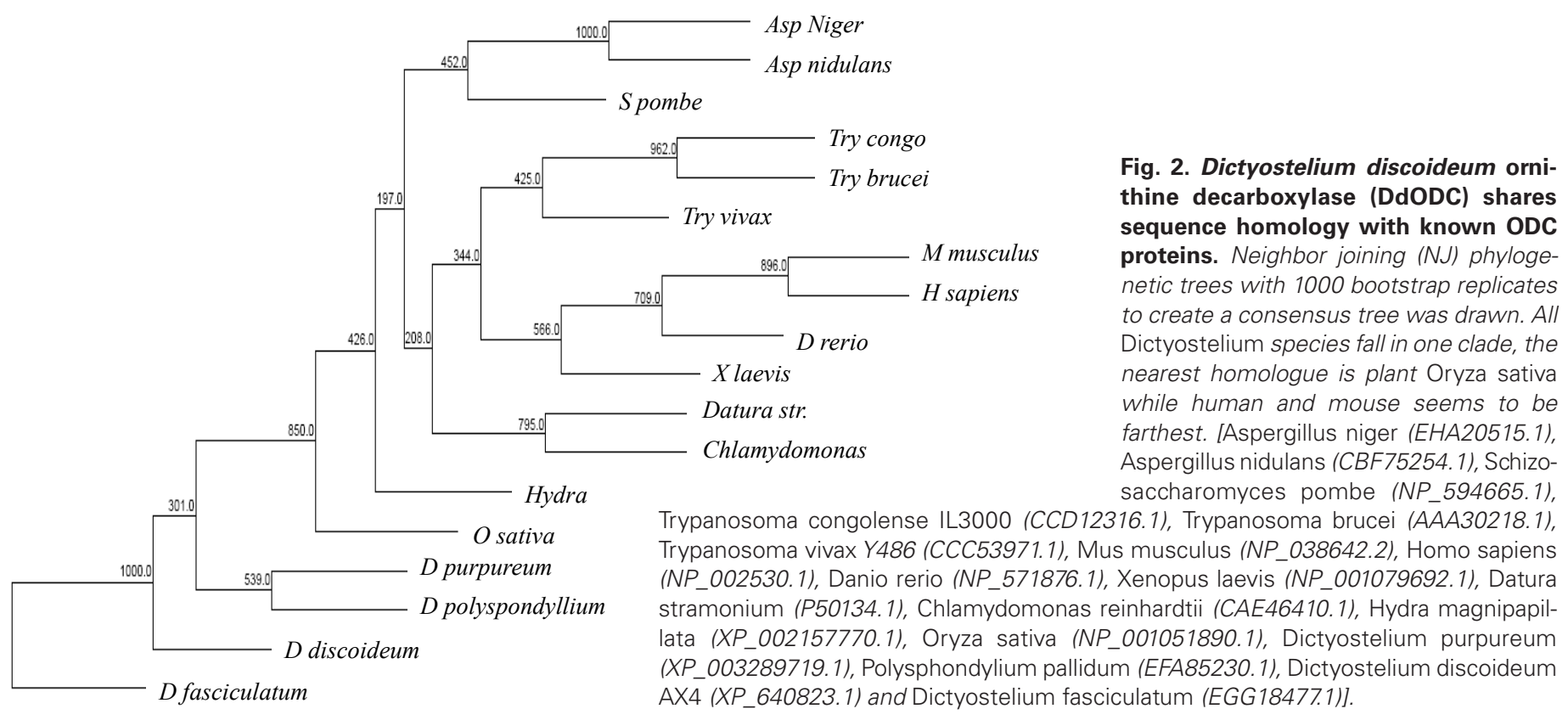


as these processes are intermingled. Here, we have chosen Dictyostelium discoideum as both growth and development could be studied independent of each other.

\section{Isolation of ODC homologue in D. discoideum}

D. discoideum ortholog of ODC (DdODC; gene id: DDB_G0281109) is located on chromosome 3 between coordinates 4069177 to 4070741 . Based on amino acid sequence, the putative DdODC showed $49 \%$ similarity with Xenopus laevis, $45 \%$ with humans and $43 \%$ with Aspergillus niger. It has 3 exons and two introns with the genomic DNA length being $1565 \mathrm{bp}$. It is a single copy gene coding for 461 amino acids having a calculated mass of $51.7 \mathrm{kDa}$.

Domain architecture analysis shows the presence of two domains (Fig. 1A); ornithine and arginine decarboxylase (Orn_Arg_deC-N; 91-325 aa) and ornithine decarboxylase $\mathrm{C}$-terminal sheet domain (Orn_DAP_Arg_deC; 328-450 aa). They are collectively known as group IV decarboxylases which are pyridoxaldependent and act on ornithine, lysine and arginine. Sequences required for the catalytic activity and dimerization in human ODC (HsODC) are also found in DdODC. Similarly, the conserved lysine-69 residue required for attachment of pyridoxal-phosphate group and the stretch of three consecutive glycine residues proposed to participate in substrate-binding in HsODC (Fig. 1B) are also found. Glycine-387 residue essential for dimerization of monomers in $\mathrm{HsODC}$ is present at position 432. Sequence motif PFYAVKCN corresponding to position $64-71$ of mammalian ODC containing lysine-69 residue is present at position 111-118 in DdODC except that phenylalanine is changed to tyrosine. Consensus sequence GPTCDGLD present in various eukaryotes is also found in DdODC (position 400-408) but has serine and isoleucine instead of glycine and leucine. Cysteine residue responsible for binding to difluoromethylornithine (DFMO) is present. Other conserved signature sequences/motifs like D(I/V)GGGF, FDCAS, EPGR, FNGF, and GAYT are also found though their functional significance is still unknown.

Evolutionary conservation analyses (Fig. 2) show all Dictyostelium species to
A
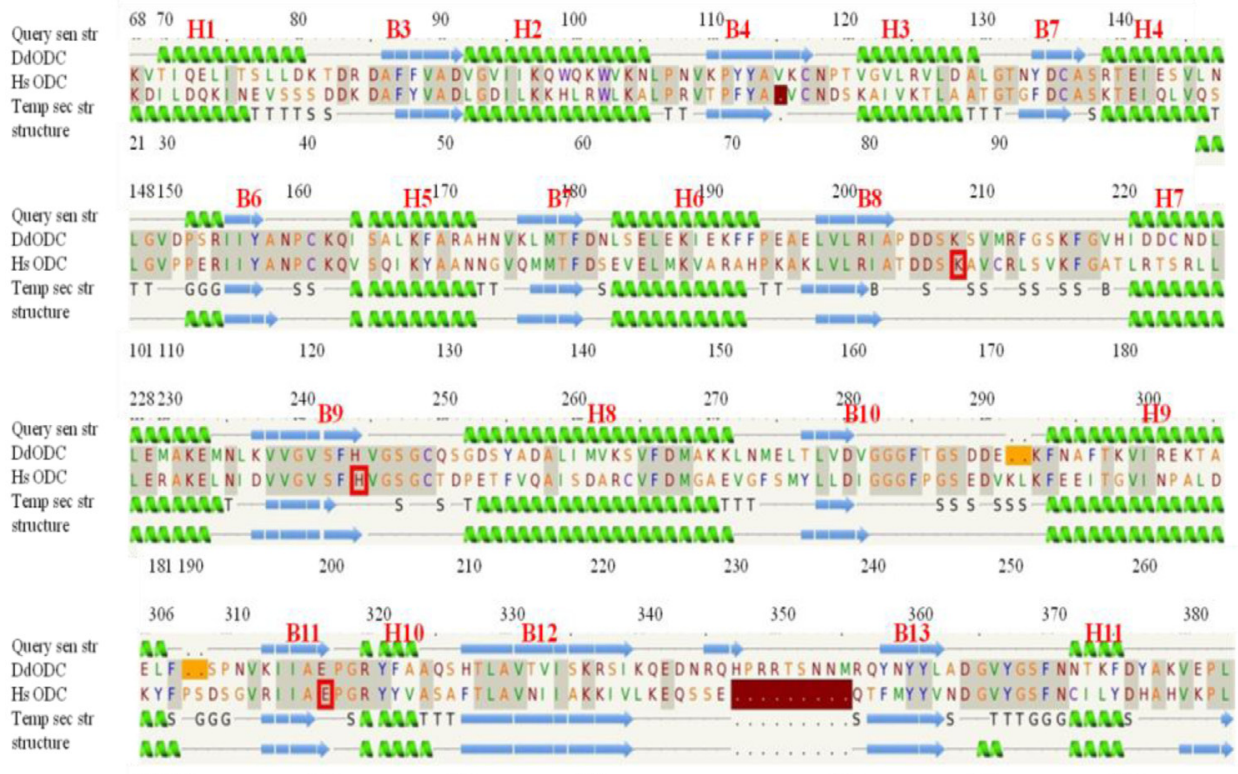

Temp sec str
structure

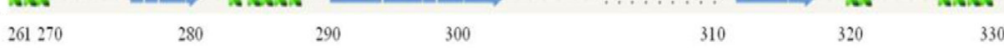

Query sen str
DdODC

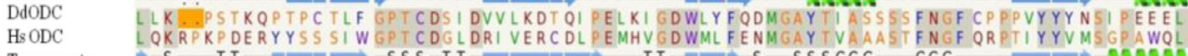

Temp sec str

structure

340

350

360

370

380

390

400

410
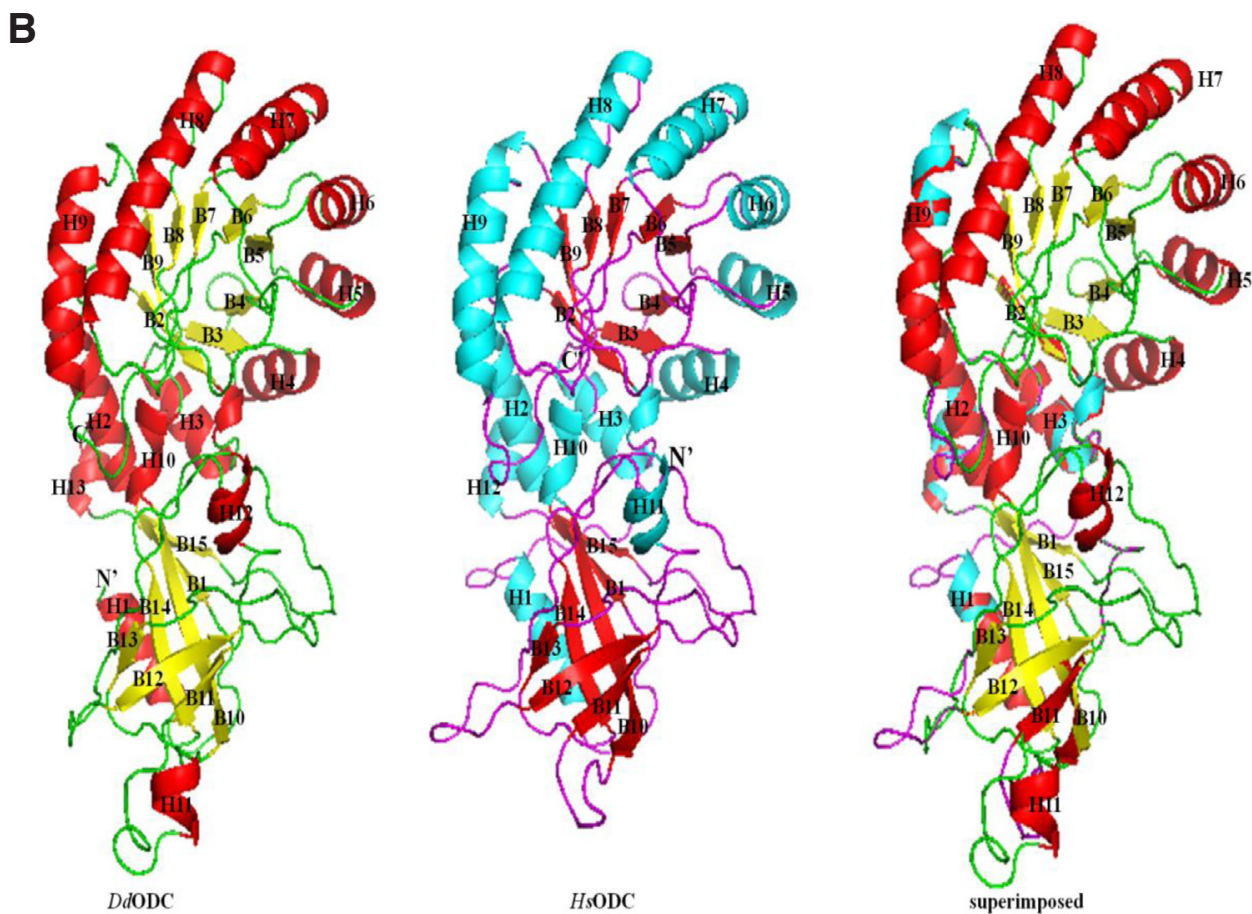

Fig. 3. Tertiary structure prediction of putative Dictyostelium discoideum ornithine decarboxylase (DdODC). (A) Primary sequence alignment between human (HsODC) and D. discoideum (DdODC) ODCs. Secondary structure elements are labeled above the primary sequence; $\alpha$-helices are identified with $H$, while $\beta$-strands are labeled with B. Numbering is based on HsODC. Secondary structure of DdODC was predicted by phyre2 software and compared to HsODC which showed best homology. (B) Tertiary structure of ODCs from Dictyostelium, human and superimposed structure of the same. 
A
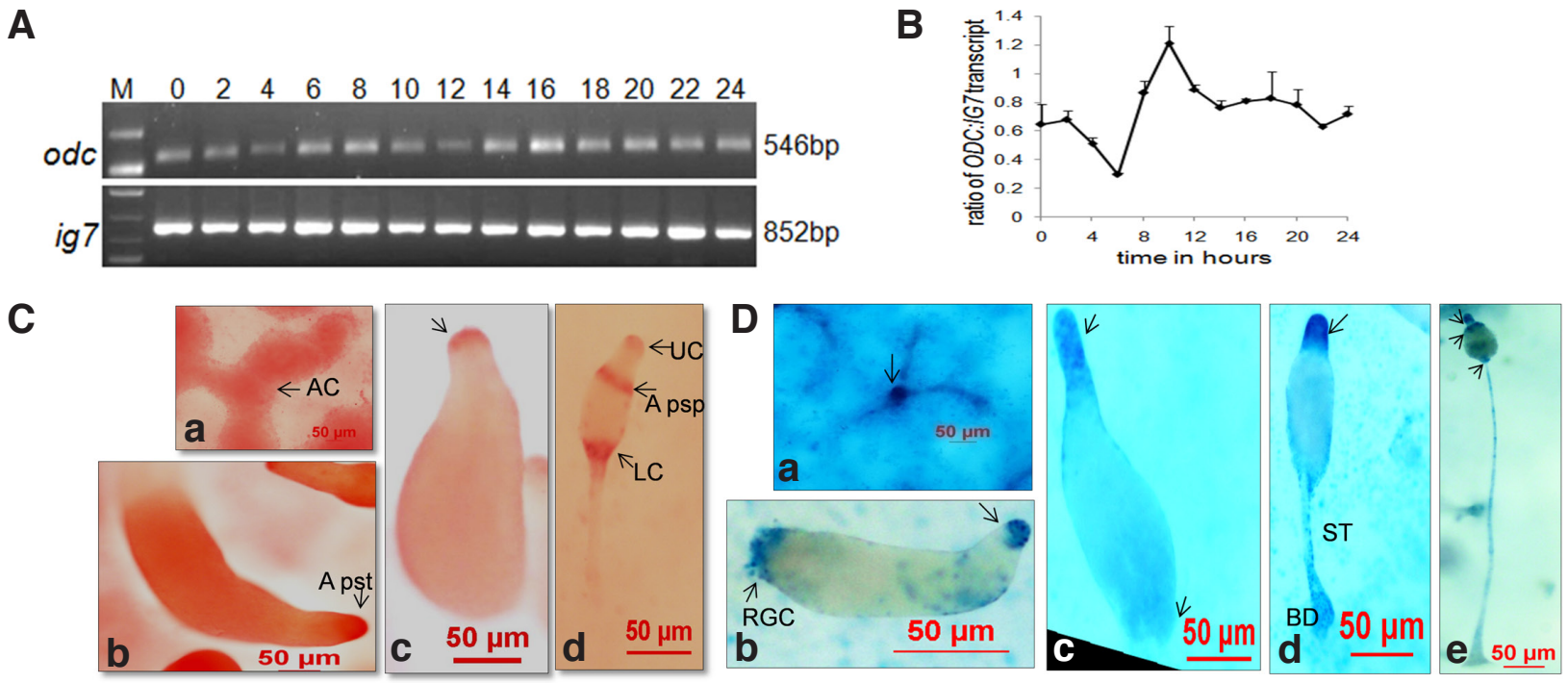

Fig. 4. Spatio-temporal RNA expression pattern of Dictyostelium discoideum ornithine decarboxylase (DdODC). (A) Temporal expression pattern of Ddodc and its comparison to controlig7 as studied by RT-PCR. Time after starvation is indicated. (B) Relative expression as observed in (A) [ $\mathrm{n}=3]$. (C) In situ hybridization by DIG-labeled Ddodc RNA probe. (D) Spatial expression pattern of putative odc promoter as detected by ubi-LacZ. Abbreviations: M, marker; a, aggregate; $b$, slug; $c$, early culminant; $d$, mid culminant; e, fruiting body; Ac, aggregate centre; Apst, anterior prestalk; UC, upper cup; LC, lower cup; Apsp, anterior prespore; RGC, rear guard cells; st, stalk; BD, basal disc).

fall close to each other. The nearest homologue is the plant Oryza sativa while human and mouse sequences seem to be farthest.

\section{Homology modeling}

A comparative protein structure model was generated to study the degree of structural resemblance. Secondary and tertiary structures of the candidate protein were predicted by phyre2 (http://www.sbg.bio.ac.uk.phyre2) prediction server. Best homologous HsODC protein structure (c1d7kB) was used as template.
Numbering of helices and sheets on the secondary structure (Fig. $3 \mathrm{~A}$ ) is based on that observed with HsODC (Almrud et al., 2000). The homology was found to be nearly $100 \%$. First two $\beta$-sheets, possibly present between amino acid 1 to 67 are not observed. A $\beta$-sheet comprising of 3 amino acid between $\beta$-sheets 12 and 13 is present in DdODC. Accordingly, $\beta 14$-sheet present in HsODC comprising of 2 amino acid is absent in DdODC. High sequence homology and secondary structure prediction suggests that our query protein possibly is ODC which is also validated based on
A

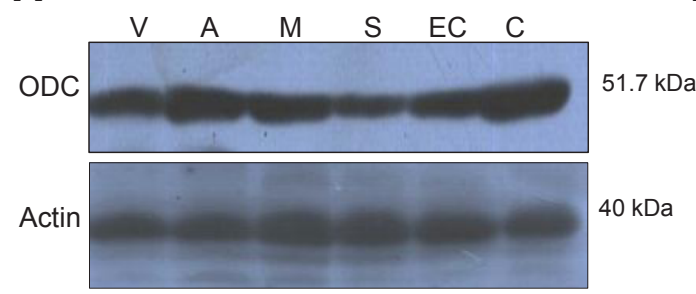

C

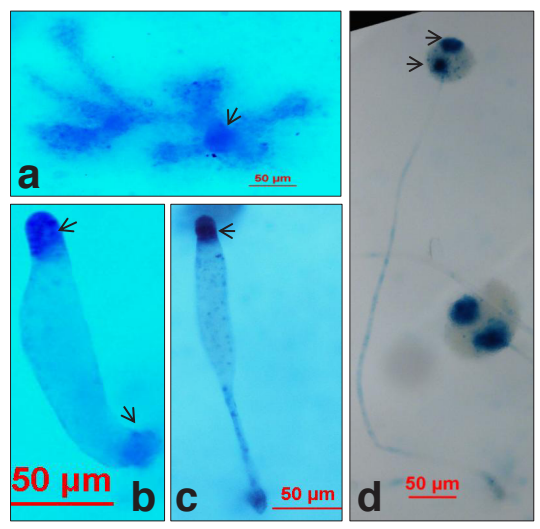

B
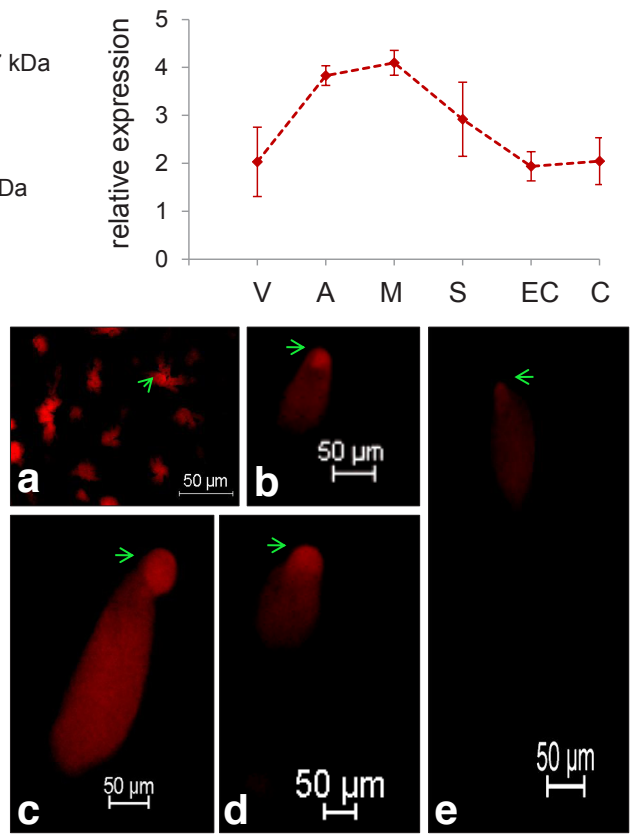

Fig. 5. Spatio-temporal protein expression pattern of Dictyostelium discoideum ornithine decarboxylase (DdODC). (A) Temporal expression pattern of DdODC and its comparison to control actin as studied by Western hybridization. (B) Relative expression as observed in (A) $[n=5]$. (C) Spatial expression pattern of ODC fusion protein as studied in Ax2/[odc]:odc-LacZ. (D) Immunofluorescence analysis in multicellular structures as studied using antibody against ODC. ( $V$, vegetative; $A$, aggregate; $M$, mound; $S$, slug; $E C$, early culminant, $C$, culminant). 
free energy (Supplementary 1).

Predicted structure showed a confidence level of nearly $100 \%$. Stereochemistry of the model prepared for full DdODC protein (Fig. $3 \mathrm{~B}$ ) is in agreement with most of the residues located in most favoured and additionally allowed regions of the Ramachandran plot (Supplementary 2). Residues present in the favoured regions are $90.1 \%$ and residues in the additional allowed region is $8.9 \% .1 .0 \%$ residues are in generously allowed region and $0 \%$ residues are in disallowed regions in the Ramachandran plot.

\section{Expression analysis of DdODC}

Expression of encoded mRNA and activity of the translated protein was found at all stages of development with higher levels during mound $\left(\mathrm{t}_{10}\right)$ (Figs. 4 and 5). RNA expression as studied by RT-PCR (Figs. $4 \mathrm{~A}$ and $\mathrm{B}$ ), in situ hybridization (Fig. 4C) and fusion of $\mathrm{LacZ}$ to putative odc promoter (Fig. 4D), was found at all stages of development and in both cell types with higher levels in prestalk/stalk cells. Expression was high in slug tips corresponding to EcmA (anterior-most prestalk) cells and in fruiting body we found expression both in upper and lower cups and in anterior-most spore region. Minor discrepancies observed with results obtained in Fig. $4 C$ and $D$ could be attributed to the kind of products being visualized in both the cases.

Western analysis revealed similar results (Fig. 5 A,B). Protein expression as revealed by expression of ORF under its own promoter showed expression at all stages of development and higher levels in prestalk/stalk cells (Fig. 5C). Immunocytochemistry data revealed similar results (Fig. 5D).

\section{Overexpression of DdODC inhibits cell proliferation and leads to mild developmental defects}

Full length odcORF was expressed under a constitutive promoter actin15as a fusion with enhanced yellow fluorescent protein (Eyfp) at C-terminal to make (Ax2[act15]:odc-Eyfp, (ODC $\left.{ }^{\circ}\right)$. The level of putrescine increased nearly 1.7 fold as compared to wild-type (data not shown). Eyfp (green) tagged DdODC was cytoplasmic with predominant intense perinuclear staining (Fig. 6A) showing high expression in vesicle like structure close to the nucleus and is in agreement with majority of earlier studies (Schipper and Verhofstad 2002). Notably, the perinuclear signal was very strong and did not overlap with lysotracker staining (data not shown). Role of ODC in the cytoplasm still needs to be elucidated but various studies indicate that polyamines may play essential roles in protein biosynthesis. Further analysis is still required to characterize the functions of ODC during cell-type differentiation.

ODC ${ }^{\mathrm{OE}}$ cells grow slow and do not show a sharp decline phase (Fig. 6B). Development too was slow with mounds being formed at $8 \mathrm{~h}$ after starvation as compared to $6 \mathrm{~h}$ in case of wild-type. Slug formed at $18 \mathrm{~h}$ as compared to $12 \mathrm{~h}$ for wild-type. Maximum delay
A

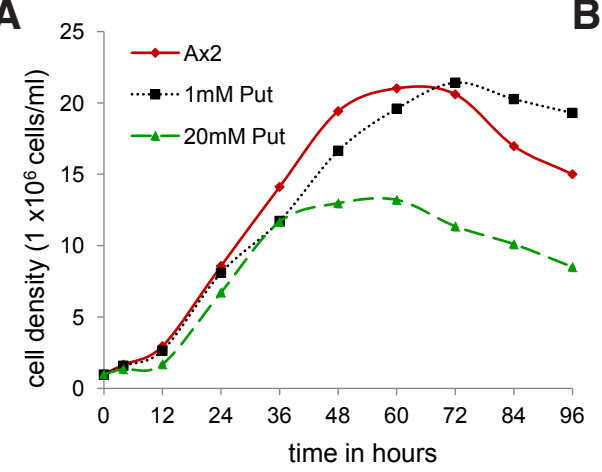

B

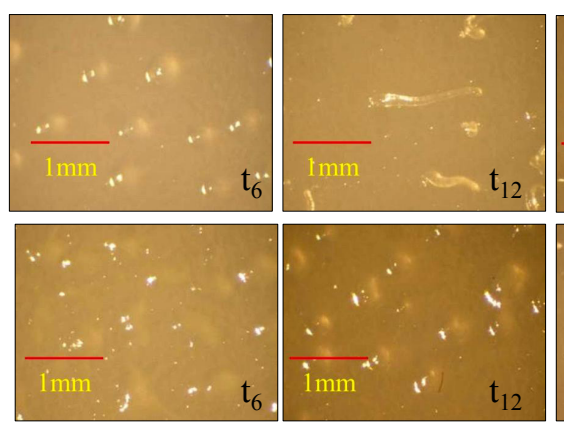

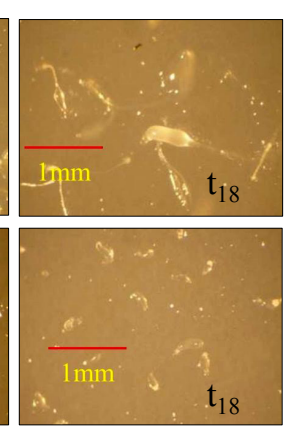

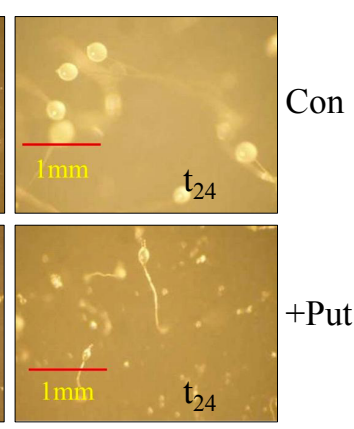

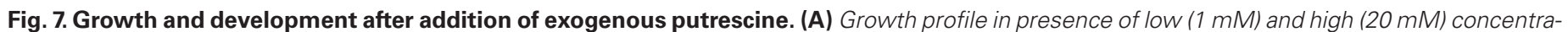
tions of putrescine. (B) Development profiles of wild-type and putrescine treated cells developed in the absence of putrescine. [n=4-5]. 

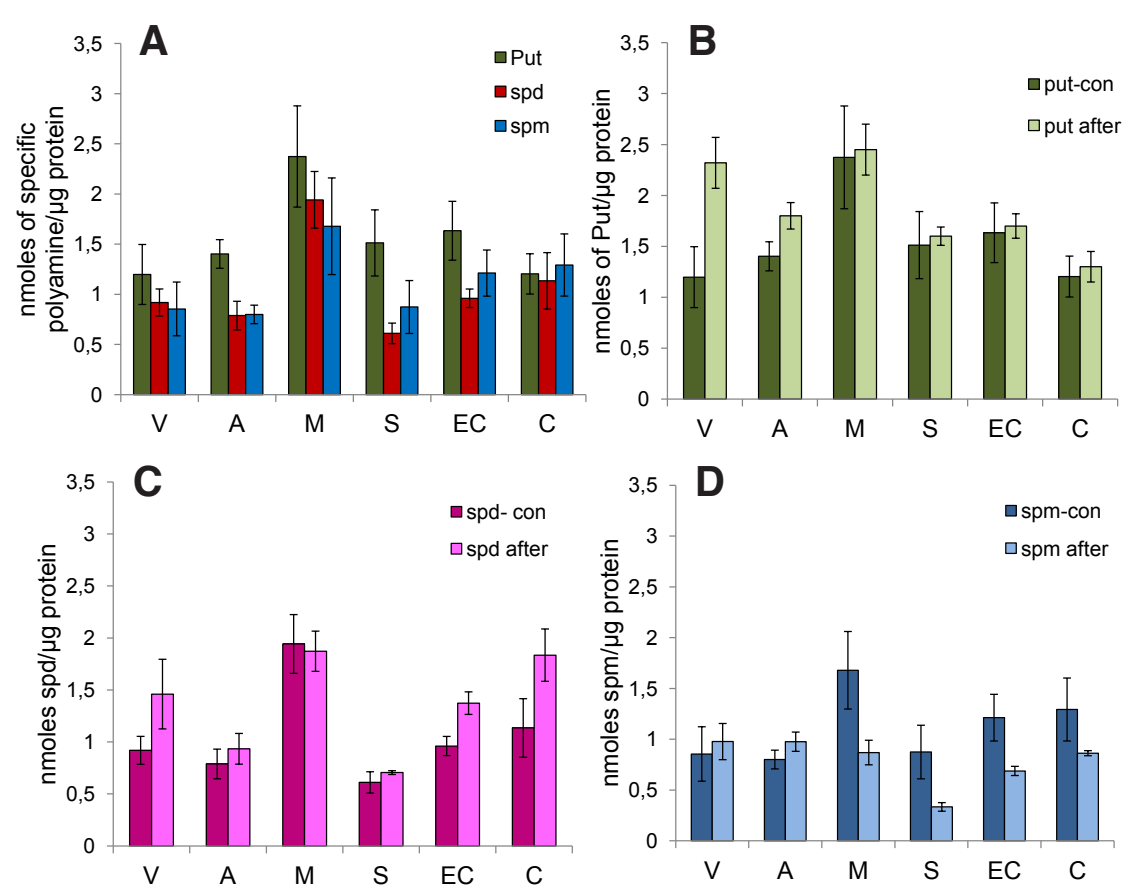

Fig. 8. Levels of various polyamines in control and after putrescine treatment during development. (A) Levels of putrescine (Put), spermidine (Spd) and spermine (Spm) during development. (B) Levels of putrescine (C) spermidine and (D) spermine in control and after putrescine treatment during development. Cells were treated for 48 hours with $20 \mathrm{mM}$ putrescine and allowed to develop in the absence of putrescine. $(V$, vegetative; $A$, aggregate; $M$, mound; $S$, slug; $E C$, early culminant; $C$, culminant). [ $n=6$ ].

was in forming slugs but time taken to form fruiting bodies was far less. Additionally, number of aggregates and sizes of the multicellular structures formed were smaller in comparison to wild-type (Fig. 6C).

Polyamines are essential for promoting cell growth butexcessive accumulation of putrescine provokes apoptosis (Takao et al., 2006). In case of $D$. discoideum, there is no apoptosis but autophagic cell death occurs during stalk cell formation (Swer et al., 2014a, b). Polyamines increase $\mathrm{Ca}^{2+}$ accumulation in mitochondria; modulate mitochondrial permeability transition, and trigger cell death. In addition, elevation of cytoplasmic $\mathrm{Ca}^{2+}$ can lead to production of reactive oxygen species and vice-versa (Minois et al., 2011). Oxidative stress could disrupt calcium homeostasis by promoting membrane lipid peroxidation and covalent modification of transporters (Jain and Shohet 1981; Kaneko et al., 1994). Both oxidative stress and disruption of $\mathrm{Ca}^{2+}$ homeostasis appear to contribute to autophagy (Swer et al., 2014b). We still need a clear understanding of calcium, ODC levels in the dying population which undergo autophagy in $D$. discoideum.

\section{Exogenous putrescine inhibits growth}

To address if the observed developmental defects were due to an increase in putrescine levels or of any other polyamines, we treated the cells with exogenous putrescine and monitored its effect both on growth and development. Lower concentrations of putrescine did not show any significant change in growth profile but at higher concentrations effect was similar to that observed with ODC ${ }^{O E}$ (Fig. 7A). Development had slowed down till slug formation taking nearly $6 \mathrm{~h}$ extra in comparison to wild-type (Fig.
7B). Henceforth, development was near normal. Putrescine treatment formed smaller multicellular structures. Our unpublished results suggest that spermidine $(4 \mathrm{mM})$ and spermine $(0.35 \mathrm{mM})$ treatments further reduced the size and number of multicellular structures in comparison to putrescine treatment. Sizes of multicellular structures formed decreased as control>putrescine>sper midine>spermine treatments. Results confirmed that high putrescine levels were detrimental for cell proliferation in $D$. discoideum and brought about small developmental defects in comparison to other polyamines.

During normal development, high level of putrescine followed by spermidine and spermine at all stages of development is observed. Levels of all three were found to be highest at mound stage which coincides with the timing of initiation of cell-type gene expression. Level of spermine marginally increased over that of spermidine from migrating slug onwards, while the level of putrescine comparatively dropped reaching to near equal amounts by the time of culmination (Fig. 8A). To confirm if putrescine levels were important for the observed developmental defects, we treated the cells with exogenous putrescine $(20 \mathrm{mM})$ for $48 \mathrm{~h}$ and measured the levels of various polyamines at specific developmental stages and compared them to that observed with wild-type (Fig. 8). Results show nearly two-fold increase in putrescine levels in freshly starved cells (V), thereafter, levels decreased to control levels (Fig. 8B). An initial increase in the level of spermidine was observed and later a gradual increase over control after slug stage (Fig. 8C) was seen. Effect was just opposite for spermine levels (Fig. 8D) where we found a decrease over the control after mound stage till culmination. Results, thus confirmed putrescine to be largely involved in cell proliferation while spermine and spermidine in controlling development which prompted us to analyze their behaviour during cell-cycle progression (Fig. 9). The precise mechanism by which putrescine show growth inhibition remains to be elucidated but possibly by accumulation in G2/M phase of cell cycle. It is known that the expression profile of both, polyamines and cyclins/cdks show changes through cell-cycle but

\section{TABLE 1}

\section{OLIGOS USED IN THIS STUDY}

\begin{tabular}{|c|c|c|}
\hline set & name & Oligo: $5^{\prime}$ to $3^{\prime}$ \\
\hline 1. & In situ & $\begin{array}{l}\text { FP-HindIII-5'-CATGAAGCTTTAGTATTAAGAATTGCACCAGATG -3' } \\
\text { RP-BamHI-5'-AGATGGATCCGTAATAATAAACTGGTGGTGGACA -3' }\end{array}$ \\
\hline 2. & RT-PCR-odc & $\begin{array}{l}\text { FP-5'- GATGCGGATCCACAAAAAGAAATGGTGAAGAAGTTG-3' } \\
\text { RP-5'- GATCGGTACCTTCACTAAGATTATCAAATGTCAT -3'. }\end{array}$ \\
\hline 3. & RT-PCR-ig7 & $\begin{array}{l}\text { FP-5'-GGATTCTGCAAAATGGCAAC-3' } \\
\text { RP- 5'-GTCCTCTCGTACTAAAGGAAGG-3' }\end{array}$ \\
\hline 4. & $\mathrm{ODC}^{\mathrm{OE}}$ & $\begin{array}{l}\text { FP:(BamHI):5'- } \\
\text { ACGCGGATCCAATCAATCTAAAACTATAAATTTAATATATAATG-3' } \\
\text { RP:(XhoI):5'- } \\
\text { ACTACTCGAGTATAACCAATTGAAAAACCTGAAATTATATGAG-3' }\end{array}$ \\
\hline 5. & ODC-P & $\begin{array}{l}\text { FP-XbaI- 5'- AGTCTCTAGAATAGATCAACCATTGAAATCACTTG -3' } \\
\text { RP-BgIII- 5'- AGTCAGATCTGGTAGATTCAGTGGTTTCAGTTG -3' }\end{array}$ \\
\hline 6. & ODC-P-ORF & $\begin{array}{l}\text { FP-BglII 5'- CTGAAGATCTCAATTAAATTTCCAAAAATGAGC-3' } \\
\text { RP-BgIII 5'-ACTAAGATCTATAACCAATTGAAAAACCTGAAATTATATG-3' }\end{array}$ \\
\hline
\end{tabular}




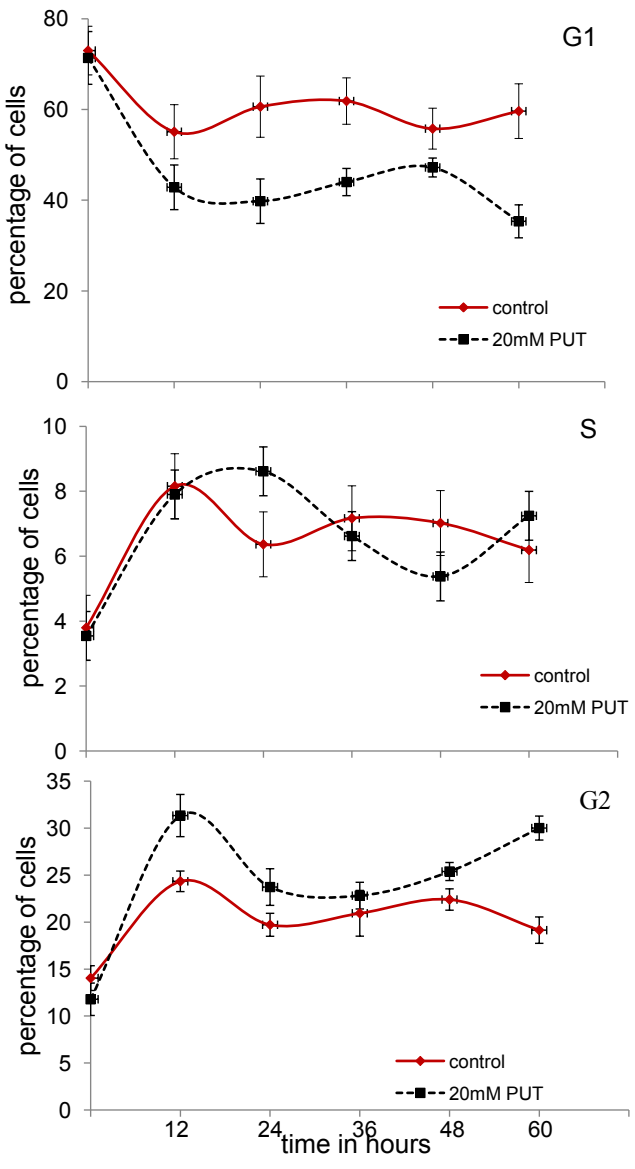

Fig. 9. Cell cycle analysis after addition of exogenous putrescine. Percentage of cells in each phase of cell-cycle in treated and control cells. [ $n=4]$.

their interaction remains to be defined.

In conclusion, we identified, cloned and characterized a novel ODC from $D$. discoideum genome. ODC expression was found at all stages of development with highest during initiation of differentiation. It was largely expressed in the dying cell population. We report that accumulation of putrescine inhibits cell proliferation but changes in developmental pattern are largely due to effective changes in spermidine and spermine levels. Our findings here advance our understanding of DdODC at the molecular level and permits future work in this area. It would be interesting to find the correlation between polyamines, calcium and ROS in bringing about autophagy in this organism.

\section{Materials and Methods}

\section{Cell culture, growth and development}

$D$. discoideum, Ax2 (axenic strain) cells (unicellular) were grown and developed according to Gosain et al., (2012). The medium was supplemented with antibiotics as indicated.

\section{Bioinformatic analyses of DdODC}

Genomic DNA, cDNA and protein sequences of DdODC were obtained from dictyBase online resource (http://www.dictybase.org). Domain architecture of DdODC protein was deduced by Simple Modular Architecture Research Tool (http://SMART.embl-heidelberg.de). ODC orthologues were searched by Basic Local Alignment Search Tool (BLASTp) at NCBI (http://
blast.ncbi.nlm.nih.gov/Blast.cgi), UniProt and dictyBase. Multiple alignments were performed using ClustalW2 at EBI server ((http://www.ebi. ac.uk/Tools/clustalw2/). PHYLIP package (Phylogeny Inference Package, version 3.68) was used to construct Neighbor joining (NJ) phylogenetic trees with 1000 bootstrap replicates to create a consensus tree.

\section{RNA detection by in situ hybridization and RT-PCR}

In situ hybridization: It was carried by the method of Gosain et al., (2012). Probes were obtained by in vitro transcription of exonic region after cloning in commercially available pBluescriptll phagemid vector. RNA (genomic position 772-1529 bases) was synthesized from exonic region amplified by PCR using the primers set1 (Table 1) and cloned into HindIII/ BamHI site of vector (supplementary 3 ). Construct pBSIISK+ (odc probe) was digested with Hindlll to yield template for antisense probe synthesis by T3 RNA polymerase, while digestion with BamHI yield template for sense probe synthesis by T7 RNA polymerase. Sense and antisense probes were processed for hydrolysis due to their larger size $(0.757 \mathrm{~kb})$.

$R T-P C R$ : This was carried out by the method described in Gosain et al., (2012). RT-PCR reactions were performed using gene specific primer set2 for odc and set3 for ig7 (internal control) (Table1).

\section{Protein detection by Western hybridization and immunocytochem- istry}

Western hybridization was essentially carried out as given in Swer et al., (2014b) while immunocytochemistry was performed according to Alvarez-Curto et al., (2007). Anti-ODC1 (\#HPA001536) and anti-actin (\#04-1040) antibodies were purchased from Sigma. The HRP conjugated secondary antibody was from Chemicon International (\#AP307P) and was detected with Supersignal chemoluminescence kit (Pierce, USA) according to the manufacturer's instructions while the TRITC conjugated secondary antibody was from Sigma (\#T6678).

\section{Plasmid construction and transformation}

p[act15]:odc-Eyfp: Ddodc gene was PCR amplified using primer set4 (Table1) from genomic DNA and constitutively expressed under actin15 as a fusion protein with Eyfp at C-terminal (Gosain et al., 2012).

$\mathrm{p}[$ odc]: ubi-LacZ: 1104bp odc intergenic region from genomic DNA was PCR amplified using primer set5 (Table1). Product was digested with Xbal/ $\mathrm{BgIll}$ and ligated into vector upstream and in frame with ubi-LacZ open reading frame (ORF) sequence of $\mathrm{p} E c m A O / 63 u b i-L a c Z$ vector, replacing ecmAO promoter to drive expressions of ubi-LacZ (ubiquitinated ile-gal).

$\mathrm{p}[$ odc]:odc:LacZ: Putative promoter with ORF region $(2.51 \mathrm{~Kb})$ of $D$ dodc was PCR amplified using primer set6. PCR product and $p E c m A O / L a c Z$ vector were digested with Bglll and ligated in a non-directional cloning, replacing ecmAO promoter. Direction of insert was confirmed by internal digestion with Xbal which is present at $963 \mathrm{bp}$. Vectors were individually transformed in Ax2 cells and selected with $100 \mu \mathrm{g} / \mathrm{mL}$ of G418.

\section{$X-g a l$ staining of developing structures}

Transformants were developed on treated dialysis membranes and processed for $\beta$-galactosidase staining as given in Swer et al., (2014a).

\section{Measurement of various polyamine levels}

Polyamine was estimated according to Saran (1998) with slight modifications. Briefly, portions of perchloric acid extracts were dansylated with an equal volume of dansyl chloride $(5 \mathrm{mg} / \mathrm{mL}$ of acetone) in presence of saturating $\mathrm{Na}_{2} \mathrm{CO}_{3}$. Excess dansyl chloride was removed by addition of proline ( $1 \mathrm{~g} / \mathrm{mL}$ of water) and incubated in dark. Mixture was then extracted with benzene and the organic phase was collected and loaded on TLC plates. Chromatogram was developed with cyclohexane: ethyl acetate $(5: 4, v / v)$, dried, visualized and photographed using excitation wavelength $366 \mathrm{~nm}$ and emission wavelength $495 \mathrm{~nm}$. Spots developed were quantified using spot densitometry program of Alpha imager. Values were obtained using standard curve prepared with different concentrations of mix and calculated on the basis of protein content. 
Cell cycle analysis: Cell cycle analysis was carried out according to the protocol followed by Swer et al., (2014b).

\section{References}

ALMRUD JJ, OLIVEIRA MA, KERN AD, GRISHIN NV, PHILLIPS MA, HACKERT ML (2000). Crystal structure of human ornithine decarboxylase at 2.1 A resolution: structural insights to antizyme binding. $J$ Mol Biol 295: 7-16.

ALVAREZ-CURTO E, SARAN S, MEIMAM, ZOBEL J, SCOTT C, SCHAAP P (2007). cAMP production by adenylyl cyclase $\mathrm{G}$ induces prespore differentiation in Dictyostelium slugs. Development 134: 959-966.

BONNER JT (1944). A discriptive study on the development of the slime mold Dictyostelium discoideum. Am J Bot 31: 175-182.

CAMPAGNE VAN LOOKEREN MM, LÖWIK CG (1985). The developmental regulation of L-ornithine decarboxylase in Dictyostelium discoideum and its induction by cAMP. Biochim Biophys Acta 846: 55-63.

EISENBERG T, KNAUER H, SCHAUER A, BÜTTNER S, RUCKENSTUHL C, CARMONA-GUTIERREZ D, RING J, SCHROEDER S, et al., (2009). Induction of autophagy by spermidine promotes longevity. Nat Cell Biol 11: 1305-1314.

FILLINGAME RH, JORSTAD CM, MORRIS DR (1975). Increased cellular levels of spermidine or spermine are required for optimal DNA synthesis in lymphocytes activated by concanavalin A. Proc Natl Acad Sci USA 72: 4042-4045.

GOSAINA, LOHIAR, SHRIVASTAVAA, SARANS (2012). Identification and characterization of peptide: $\mathrm{N}$-glycanase from Dictyostelium discoideum. BMC Biochem 13:9.

HARRIS WA, NORTH MJ (1982). Osmotically induced changes in the ornithine decarboxylase activity of Dictyostelium discoideum. J Bact 150: 716-721.

HEBYO, PERSSON L (1990). Molecular genetics of polyamine synthesis in eukaryotic cells. Trends Biochem Sci 15: 153-158.

HELLER JS, FONG WF, CANELLAKIS ES (1976). Induction of a protein inhibitor to ornithine decarboxylase by the end products of its reaction. Proc Natl Acad Sci
USA 73: 1858-1862.

JAIN SK, SHOHET SB (1981). Calcium potentiates the peroxidation of erythrocyte membrane lipids. Biochim Biophys Acta 642: 46-54.

JÄNNE J, ALHONEN L, PIETILÄ M, KEINÄNEN TA (2004). Genetic approaches to the cellular functions of polyamines in mammals. Eur $J$ Biochem 271: 877-894.

KANEKO M, MATSUMOTO Y, HAYASHI H, KOBAYASHI A, YAMAZAKI N (1994). Oxygen free radicals and calcium homeostasis in the heart. Mol Cell Biochem 139: 9-100.

MINOIS N, CARMONA-GUTIERREZ D, MADEO F (2011). Polyamines in aging and disease. Aging (Albany NY) 3: 716-732.

PEGG AE, MCCANN PP (1982). Polyamine metabolism and function. Am J Physiol 243: C212-221.

SARAN S (1998). Changes in endogenous polyamine levels are associated with differentiation in Dictyostelium discoideum. Cell Biol Int 22: 575-580.

SCHIPPER RG, VERHOFSTAD AAJ (2002). Distribution patterns of ornithine decarboxylase in cells and tissues: facts, problems, and postulates. $J$ Histochem Cytochem 50: 1143-1160.

SWER PB, BHADORIYA P, SARAN S (2014a). Analysis of Rheb in the cellular slime mold Dictyostelium discoideum: cellular localization, spatial expression and overexpression. J Biosci 39: 75-84.

SWER PB, LOHIA R, SARAN S (2014b). Analysis of rapamycin induced autophagy in Dictyostelium discoideum. Indian J Exp Biol 52: 295-304.

TABOR CW, TABOR H (1976). 1,4-Diaminobutane (putrescine), spermidine, and spermine. Annu Rev Biochem 45: 285-306.

TABORCW, TABORH (1985). Polyamines in microorganisms. Microbiol Rev49:81-99.

TAKAO K, RICKHAG M, HEGARDT C, OREDSSON S, PERSSON L (2006). Induction of apoptotic cell death by putrescine. Int J Biochem Cell Bio/38: 621-628.

WANG SH, SHIH YL, KO WC, WEI YH, SHIH CM (2008). Cadmium-induced autophagy and apoptosis are mediated by a calcium signaling pathway. Cell Mol Life Sci 65: 3640-3652. 


\section{Further Related Reading, published previously in the Int. J. Dev. Biol.}

An orthologue of the Myelin-gene Regulatory Transcription Factor regulates Dictyostelium prestalk differentiation Hiroshi Senoo, Hong-Yu Wang, Tsuyoshi Araki, Jeffrey G. Williams and Masashi Fukuzawa Int. J. Dev. Biol. (2012) 56: 325-334

http://dx.doi.org/10.1387/ijdb.120030jw

Identification of a target for CudA, the transcription factor which directs formation of the Dictyostelium tip organiser Hong-Yu Wang and Jeffrey G. Williams Int. J. Dev. Biol. (2010) 54: 161-165

http://dx.doi.org/10.1387/ijdb.082723hw

The mob as tumor suppressor (mats1) gene is required for growth control in developing zebrafish embryos

Yuan Yuan, Shuo Lin, Zuoyan Zhu, Wenxia Zhang and Zhi-Chun Lai

Int. J. Dev. Biol. (2009) 53: 525-533

http://dx.doi.org/10.1387/ijdb.082720yy

Expression of zinc transporter family genes in Dictyostelium

Nobuya Sunaga, Meri Monna, Nao Shimada, Mai Tsukamoto and Takefumi Kawata

Int. J. Dev. Biol. (2008) 52: 377-381

http://dx.doi.org/10.1387/ijdb.072389ns

Transient expression of apoaequorin in zebrafish embryos: extending the ability to image calcium transients during later stages of development

Chris Y. Cheung, Sarah E. Webb, Anming Meng and Andrew L. Miller

Int. J. Dev. Biol. (2006) 50: 561-569

http://dx.doi.org/10.1387/ijdb.062151cc

5 yr ISI Impact Factor $(2013)=2.879$
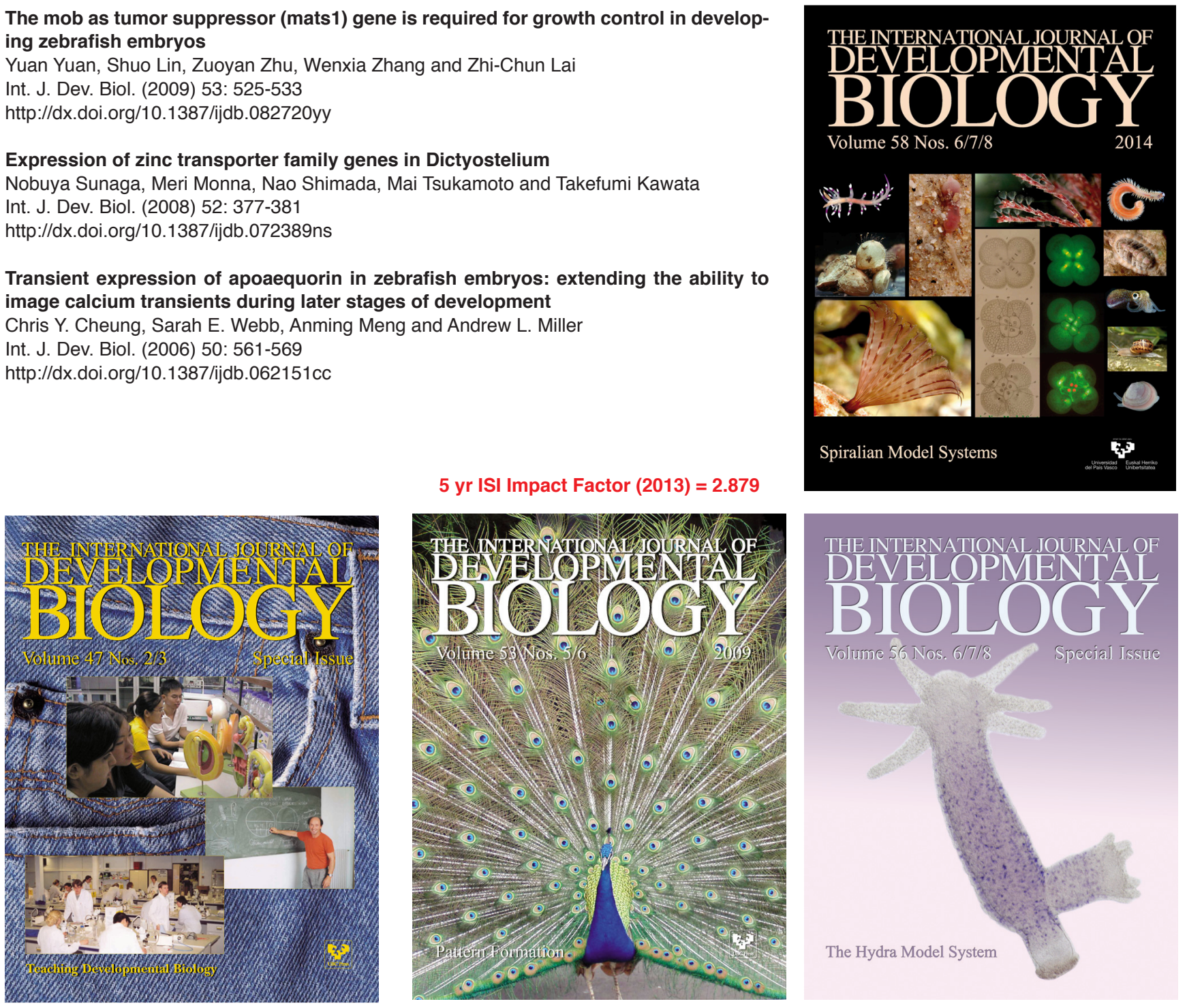